\title{
DÉCOUVERTE EN AFRIQUE OCCIDENTALE. \\ D'HUNTERELLUS HOOKERI HOWARD, PARASITE DES IXODIDÉS
}

\author{
Par c. B. PHILIP
}

Pendant mon voyage en Nigeria, comme membre de la Commission pour la fièvre jaune (Bureau international de la Fondation Rockefeller), une colonie d'Hunterellus hookeri Howard, parasite des Ixodidés, a été découverte près de Lagos. Je dois au Prof. R. A. Cooley, Bozeman, Montana, l'identification de ce chalcidien.

Il a été trouvé dans plus de 90 p. 100 de nymphes gorgées de Rhipicephalus sanguineus Latr., sur des chiens, à Apapa, au mois d'avril 1929 ; ce pourcentage est le plus élevé qui ait été rencontré.

Wood (1911) indique, comme distribution de ce parasite : le Texas et la Californie aux Etats-Unis, Monterey au Mexique et Lourenço Marques en Afrique. Da Costa Lima (1915) y ajoute le Brésil.

Un grand nombre de parasites adultes ont été observés et capturés entre les poils des mêmes chiens à Apapa pendant les mois d'avril, mai et juin. Un adulte a été vu aussi sur un chien à longs poils près de Yaba pendant le mois de février, mais il s'est si vite caché dans les poils qu'il n'a pu être capturé.

Malgré l'intensité de ce parasitisme, il est étonnant de rencontrer un si grand nombre de Rhipicephalus sur les chiens des environs de Lagos.

\section{BIBLIOGRAPHIE}

Wood H. P.). - Notes on the life history of the tick parasite Hunterellus hookeri Iloward. Journ. econ. entomol., IV, 1911, p. 425-431.

Da Costa Lima (A.). - O chalcideo Hunterellus hookeri Howard, parasita do garrapato Rhipicephalus sanguineus Latreille, observado no Rio de Janeiro. Reo. vet. zootechnia, V, 1915, p. 201-203.

Anvales de Parasitologie, $\mathrm{T}$. IX, $\mathrm{n}^{\circ} 3 .-1^{\text {er }}$ mai 1931 , p. 276. 